\title{
Magnetic Field Influence on Critical Sound Attenuation and Velocity Variation in Ferromagnets
}

\author{
A. PAWLAK \\ Faculty of Physics, Adam Mickiewicz University \\ Umultowska 85, 61-614 Poznań, Poland
}

\begin{abstract}
The ultrasound velocity and attenuation are investigated in ferromagnets under an application of magnetic field. In the magnetic field six different characteristic regimes are distinguished instead of traditional two (hydrodynamic and critical) found for the case of zero magnetic field. The mean-field Landau-Khalatnikov theory as well as the scaling predictions are given for each regime. Various critical exponents are identified for these regions and compared with the ultrasonic data for $\mathrm{MnP}$. The shift of the ultrasonic attenuation peak under the influence of magnetic field towards higher temperatures is discussed.
\end{abstract}

PACS numbers: 05.70.Jk, 62.65.+k

\section{Introduction}

The dynamics of ultrasound near the magnetic critical point is a very interesting area in which one can test modern concepts of the phase transition theory such as the scaling or the universality of critical exponents. The sound attenuation coefficient and velocity are strongly affected by an external magnetic field. In the ferromagnets the positions of the maxima in attenuation and the minima in sound velocity depend on the magnitude and direction of the magnetic field [1]. It is known [1,2] that under the influence of magnetic field applied along the easy axis the ultrasonic attenuation peak is shifted towards higher temperatures. The scaling expressions for the sound attenuation coefficient are discussed in this paper and compared with the results of the LandauKhalatnikov (LK) theory.

\section{Ultrasonic attenuation in magnetic field}

For the ferromagnets the magnetic field is coupled to the order parameter so the non-zero magnetic field destroys the phase transition. However, still a maximum in the susceptibility is observed at a temperature $T_{\max }^{\chi}(H)$ higher than the Curie point $T_{\mathrm{C}}$ [3] of the ferromagnet. The temperature of this maximum in susceptibility increases with increasing magnetic field $H$ because according to the scaling theory

$$
\begin{aligned}
& \chi(t, H)=\frac{\partial M}{\partial H}=t^{-\gamma} f_{\chi}\left(H / t^{\Delta}\right) \\
& \quad=H^{-\gamma / \Delta} g_{\chi}\left(t H^{-1 / \Delta}\right),
\end{aligned}
$$

where $t=\frac{T-T_{\mathrm{C}}}{T_{\mathrm{C}}}$ is the reduced temperature, $\Delta=\gamma+\beta$ is the gap exponent and $f_{\chi}$ and $g_{\chi}$ are scaling functions. The susceptibility maximum can be obtained from the equation: $(\partial \chi / \partial H)_{H} \propto t^{-(\gamma+1)}\left(1-a_{2} H^{2} / t^{2 \Delta}+\right.$ $\left.a_{4} H^{4} / t^{4 \Delta}+\ldots\right)=0$, where the expansion in the powers of $H$ was used $\left(a_{2}, a_{4}, \ldots\right.$ are coefficients) and only the leading terms were left. It follows immediately from this expansion that for low magnetic fields $t_{\max }^{\chi} \propto H^{1 / \Delta}$ [4]. The height of the susceptibility peak decreases as the magnetic field increases.

It is well known that in the absence of the magnetic field, the critical behaviour of the sound attenuation coefficient is characterised by the scaling relation $[5,6]$ $\alpha(t, \omega) \propto \omega^{2} t^{-\rho_{\mathrm{s}}} f_{ \pm}(y)$, where $f_{ \pm}$is the scaling function (which can have different form above and below $T_{\mathrm{C}}$ ), $y=\omega \tau_{\mathrm{c}}^{0} t^{-z \nu}$ is the reduced frequency and $\omega$ the ultrasonic frequency; $\tau_{\mathrm{c}}^{0}$ is a bare critical relaxation time for the order parameter fluctuations and $\rho_{\mathrm{s}}$ is the critical sound attenuation exponent. In the magnets that have also metallic properties large sound attenuation critical exponents are usually measured [7] and the so-called Murata-Iro-Schwabl regime is expected [5, 6, 8, 9], where $\rho_{\mathrm{s}}=z \nu+\alpha$ can be expressed by specific-heat $(\alpha)$ and correlation length $(\nu)$ exponents and the dynamic critical exponent $z$. When the magnetic field is zero the sound attenuation maximum as well as the sound velocity minimum occur slightly below the transition temperature, i.e. at the temperature at which the reduced frequency $y_{-} \approx 1$, where the index denotes the low-temperature phase. The peak in the sound attenuation below the transition temperature is due to the static polarisation $M=\langle S(x)\rangle$ of the order parameter and it is the analogue of the Landau-Khalatnikov sound damping [10] which is the only one which contributes to $\alpha(t, \omega)$ in the mean-field theory. In magnetic fields it was observed however that the sound attenuation peak is shifted towards higher temperatures and can be located even at the temperatures much higher than $T_{\mathrm{C}}$.

It was recognised by Tachiki and Maekawa [1] that this shift of the sound attenuation maximum is due to the competition between the fluctuation term (which is the 


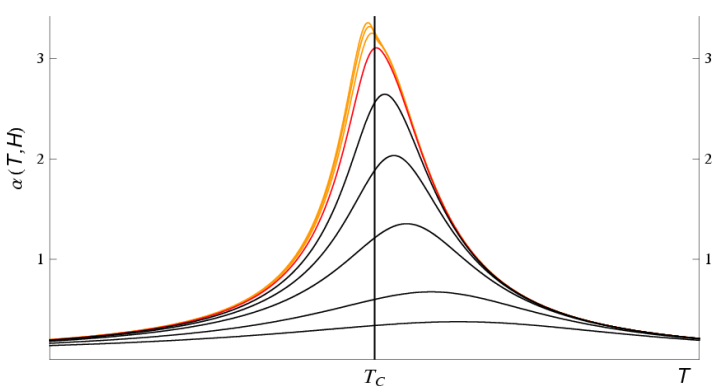

Fig. 1. The shift of the sound attenuation peak as a result of the competition between the LK term and the fluctuation contribution. The height of the peak decreases with increasing magnetic field intensity.

only one present in zero magnetic field above $T_{\mathrm{C}}$ ) and the LK term known also as the relaxational contribution, which appears whenever a static polarisation takes a non-zero value. In Fig. 1 we illustrate the competition between the LK term $\alpha(t, \omega, H)_{\mathrm{LK}} \propto \omega^{2} \frac{M(t, H)^{2} \chi(t, H)^{2}}{1+\left[\omega \tau_{\mathrm{c}}^{0} \chi(t, H)\right]^{2}}$, and the fluctuation term, which is calculated in the Gaussian approximation. The magnetisation $M(t, H)$ and the susceptibility $\chi(t, H)$ are calculated within the Landau theory e.g.

$$
\begin{gathered}
M(t, H)=\frac{-2^{1 / 3} \frac{a}{u}}{\left(27 \frac{H}{u}+\sqrt{108\left(\frac{a}{u}\right)^{3}+729\left(\frac{H}{u}\right)^{2}}\right)^{1 / 3}} \\
+\frac{\left(27 \frac{h}{u}+\sqrt{108\left(\frac{a}{u}\right)^{3}+729\left(\frac{H}{u}\right)^{2}}\right)^{1 / 3}}{3 \cdot 2^{1 / 3}},
\end{gathered}
$$

where the Landau-Ginzburg Hamiltonian has been used: $F(M)=\frac{1}{2} a M^{2}+\frac{1}{4} u M^{4}-H M$ with $a \propto T-T_{\mathrm{C}}$. It is seen in Fig. 1 that the height of the attenuation peak decreases and its location moves to the right as the magnetic field increases.

\section{Scaling}

Another challenge is the construction of the scaling theory of critical sound attenuation in an external magnetic field. In general, the sound attenuation coefficient consists of a few terms (in addition to the LK term and the fluctuation contribution there is also a mixing term $[11,12])$. These terms combine in the scaling region giving a single critical exponent and the scaling function. In other words in the scaling region there should be cancellations between different contributions and the critical sound attenuation exponent should be the same as in the disordered phase without the magnetic field $[13,12]$. Only the scaling function should include an additional field variable. Thus the scaling relation for the sound attenuation coefficient takes the form

$$
\begin{aligned}
& \alpha(t, \omega, H) \propto \omega^{2} t^{-\rho_{\mathrm{s}}} f\left(\omega t^{-z \nu}, H t^{-\Delta}\right) \quad \text { or } \\
& \alpha(t, \omega, H) \propto \omega^{2} H^{-\rho_{\mathrm{s}} / \Delta} g\left(\omega H^{-z \nu / \Delta}, t H^{-1 / \Delta}\right),
\end{aligned}
$$

where $f$ and $g$ are the sound attenuation scaling functions
$[5,6]$. This is a generalisation of the corresponding formula for the sound attenuation in zero field. The scaling functions need to be determined theoretically as well as experimentally. So far only some mean-field results have been obtained $[1,14-16]$ and the measurements far from critical temperature were carried out in $\mathrm{MnP}$ [2]. However, some results can be immediately guessed from the formulae (2) and (3). First of all, let us notice that in the case without the field the scaling functions $f_{ \pm}(y)$ tend to some constants as $y \rightarrow 0$ and $f_{ \pm}(y) \propto y^{-\rho_{\mathrm{s}}} / z \nu$ for $y \rightarrow \infty$. The last relation explains the finite value of sound attenuation coefficient at the critical point $(y=\infty)$. The range of parameter space $(t, \widetilde{\omega})$ near the critical point $(0,0)$ for which $y \ll 1$ is called the hydrodynamic range (or regime) and that for which $y \gg 1$ is called the critical range. Here $\widetilde{\omega}=\omega \tau_{\mathrm{c}}^{0}$ is a dimensionless variable which can be compared with $t^{z \nu}\left(\widetilde{\omega}=t^{z \nu}\right.$ if $\left.y=1\right)$. Addition of one variable to the parameter space induces the appearance of six instead of two (hydrodynamic and critical one) characteristic regimes in the space $(t, \widetilde{\omega}, h)$ where a dimensionless field $h$ was introduced $\left(h=H / H_{0}\right.$ with $H_{0}$ being a characteristic magnetic field of the system). They can be specified by the sequence of the leading, next to leading and the smallest variable or by the leading behaviour of $\alpha(t, \omega, H)$ and the first correction to it. Thus the six sequences or six characteristic regimes are defined by:

$$
\mathrm{H} 1: \quad \alpha \propto \omega^{2}|t|^{-\rho_{\mathrm{s}}}\left[1-a_{ \pm} \omega^{2}|t|^{-2 z \nu}+\ldots\right]
$$$$
\text { for } h^{1 / \Delta} \ll \omega^{1 / z \nu} \ll|t| \text {, }
$$$$
\mathrm{H} 2: \quad \alpha \propto \omega^{2}|t|^{-\rho_{\mathrm{s}}}\left[1+b_{ \pm} h^{2}|t|^{-2 \Delta}+\ldots\right]
$$

for $\omega^{1 / z \nu} \ll h^{1 / \Delta} \ll|t|$,

H3 : $\quad \alpha \propto \omega^{2} h^{-\rho_{\mathrm{s}} / \Delta}\left[1-c_{ \pm} \omega^{2} h^{-2 z \nu / \Delta}+\ldots\right]$

for $|t| \ll \omega^{1 / z \nu} \ll h^{1 / \Delta}$,

H4 : $\quad \alpha \propto \omega^{2} h^{-\rho_{\mathrm{s}} / \Delta}\left[1+d_{ \pm}|t| h^{-1 / \Delta}+\ldots\right]$

for $\omega^{1 / z \nu} \ll|t| \ll h^{1 / \Delta}$,

$\mathrm{C} 1: \quad \alpha \propto \omega^{2-\rho_{\mathrm{s}} / z \nu}\left[1-e_{ \pm}|t| \omega^{-1 / z \nu}+\ldots\right]$

for $|t| \ll h^{1 / \Delta} \ll \omega^{1 / z \nu}$,

$\mathrm{C} 2: \quad \alpha \propto \omega^{2-\rho_{\mathrm{s}} / z \nu}\left[1-f_{ \pm} h^{2} \omega^{-2 \Delta / z \nu}+\ldots\right]$

for $|t| \ll h^{1 / \Delta} \ll \omega^{1 / z \nu}$, where $a_{ \pm}, b_{ \pm}, \ldots$ are some constants which describe the amplitude of the first correction to the leading behaviour. The regimes $\mathrm{H} 1, \mathrm{H} 2, \ldots$ are similar to the hydrodynamic region in the case the magnetic field is zero and the regimes $\mathrm{C} 1$ and $\mathrm{C} 2$ correspond to the critical (nonhydrodynamic) behaviour. Let us illustrate these results on the example of the data obtained for $\mathrm{MnP}$ [2]. First, Eq. (H2) predicts that for very low magnetic fields and far from the critical temperature, the change in the sound attenuation is $\Delta \alpha=\alpha(0)-\alpha(h) \propto$ $\omega^{2} h^{2} t^{-\left(\rho_{\mathrm{s}}+2 \Delta\right)}$. In the mean-field approximation this relation corresponds to $\Delta \alpha \simeq \omega^{2} H^{2}|t|^{-4}$. In Fig. 2 the quadratic dependence on the magnetic field is shown. The data are from the work of Komatsubara et al. [2, 1]. For very high magnetic fields and close to the critical tem- 


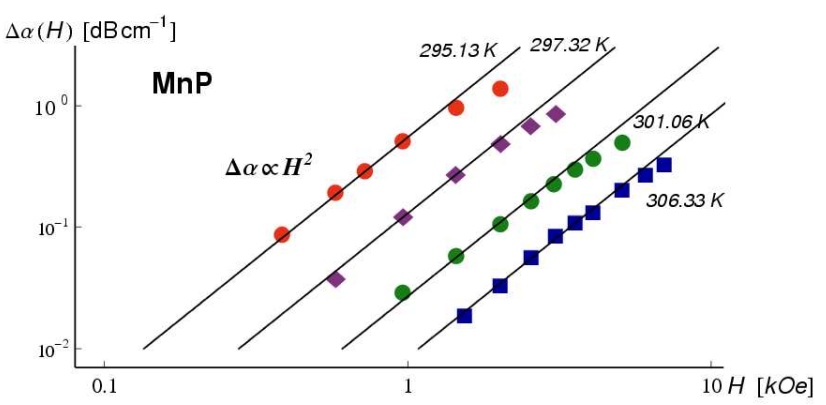

Fig. 2. The $H^{2}$ dependence of the sound attenuation change in $\mathrm{MnP}$ for the frequency $f=90 \mathrm{MHz}$. The data are taken from $[2,1]$.

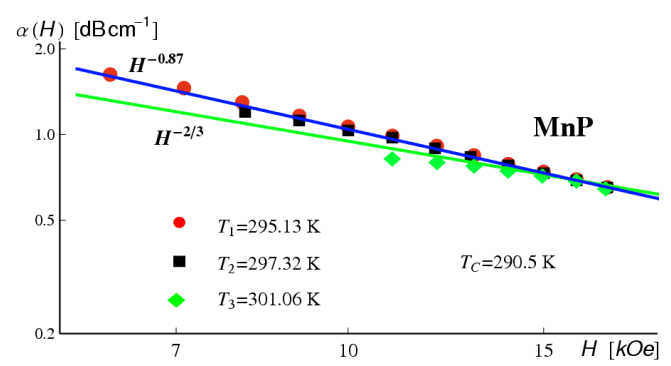

Fig. 3. The high field dependence of the longitudinal sound attenuation in $\mathrm{MnP}$ for $f=90 \mathrm{MHz}$. The data are taken from $[2,1] . T_{\mathrm{C}}=290.8 \pm 0.1 \mathrm{~K}$.

perature the attenuation scales as $\alpha(H) \propto \omega^{2} H^{-\rho_{s} / \Delta}$. In the mean-field approximation it turns into $\omega^{2} H^{-2 / 3}$. The dependence of $\alpha$ on high magnetic fields is shown in Fig. 3.

\section{Maximum in the sound attenuation coefficient}

The effect of the magnetic field on the location of the sound attenuation maximum can also be obtained. For very low magnetic field the temperature at which this maximum occurs lies below $T_{\mathrm{C}}$ and slightly increases with

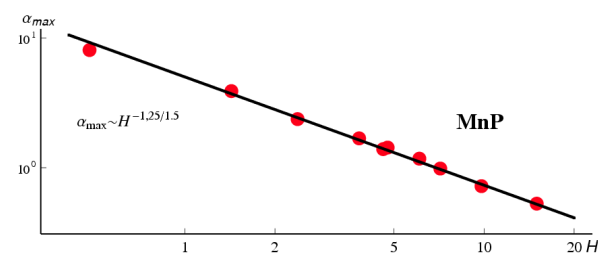

Fig. 4. The height of the ultrasonic attenuation peak as a function of the magnetic field. The data are from [2]. the increase in the magnetic field intensity: $T_{\max }-T_{\mathrm{C}} \propto$ $-\omega^{1 / z \nu}\left(1-a H^{2} \omega^{2 \Delta / z \nu}\right)$. For high magnetic fields we obtain $T_{\max }-T_{\mathrm{C}} \propto H^{1 / \Delta}\left[\left(\frac{H}{H_{1}}\right)^{\frac{z \nu}{\Delta}}-1\right]$ with $H_{1}=c \omega^{\Delta / z \nu}$. The height of this maximum is given by $\alpha_{\max } \propto H^{-z \nu / \Delta}$ which is illustrated in Fig. 4.

\section{Summary}

In this paper the competition between the LandauKhalatnikov term and the fluctuation contribution is investigated in the theory of the ultrasound attenuation in ferromagnets under application of magnetic field. Generalised scaling expressions for the sound attenuation coefficient are also given. Four novel scaling regimes are found with various critical exponents. The scaling predictions for the shift of the ultrasonic attenuation peak under the influence of the magnetic field are obtained. The theoretical results are compared with the ultrasonic data for MnP.

\section{References}

[1] M. Tachiki, S. Maekawa, Prog. Theor. Phys. 51, 1 (1974).

[2] T. Komatsubara, A. Ishizaki, S. Kusaka, E. Hirahara, Solid State Commun. 14, 741 (1974).

[3] A. Paduan-Filho, C.C. Becera, J. Magn. Magn. Mater. 261, 161 (2003).

[4] H.P. Kunkel, R.M. Roshko, G. Williams, Phys. Rev. B 37, 5880 (1988).

[5] A. Pawlak, Eur. Phys. J. B 4, 179 (1998).

[6] A. Pawlak, Sound Propagation near the Magnetic Phase Transition, Wydawnictwo Naukowe UAM, Poznań 2004 (in Polish).

[7] B. Lüthi, Physical Acoustics in the Solid State, Springer, Berlin 2005.

[8] K.K. Murata, Phys. Rev. B 13, 4015 (1976).

[9] H. Iro, F. Schwabl, Solid State Commun. 46, 205 (1983).

[10] L.D. Landau, I.M. Khalatnikov, Dokl. Akad. Nauk SSSR 96, 469 (1954); reprinted in Collected Papers of L.D. Landau, Ed. D. ter Haar, Pergamon, London 1965.

[11] J.O. Fossum, J. Phys. C 18, 5531 (1985).

[12] R. Dengler, F. Schwabl, Z. Phys B 69, 327 (1987).

[13] P.C. Hohenberg, B.I. Halperin, Rev. Mod. Phys. 49, 435 (1977).

[14] S. Maekawa, R.A. Treder, M. Tachiki, M.C. Lee, M. Levy, Phys. Rev. B 13, 1284 (1976).

[15] R. Erdem, Phys. Lett. A 312, 238 (2003).

[16] R. Erdem, M. Keskin, Phys. Lett. A 326, 27 (2004). 